\title{
The Scoop and Run Method of Pre-clinical Care for Trauma Victims
}

\author{
Shaurya Taran*
}

\section{INTRODUCTION}

Today, paramedics employ one of two different forms of treatment when providing care to trauma victims at the site of an accident. One of these practices involves administering as much medical care as possible to casualties at the trauma site itself, and is the dominant mode of pre-clinical treatment offered in most European countries. The other general approach involves providing only basic care in the field, and is the more widely employed procedure in North America. The former practice allows for a more comprehensive treatment of casualties, but also delays the arrival of a patient to a medical facility. The latter procedure, by contrast, places a greater emphasis on the speedy delivery of a casualty to the hospital. Both practices are widely used around the world, and both have their associated list of benefits and drawbacks. It is therefore difficult to name the better form of treatment, but an analysis of the success rates of both the European and North American pre-clinical practices points in favour of the latter approach. Indeed, a large body of compelling evidence suggests that patients who arrive more quickly at hospitals from accident sites fare better chances of a complete recovery than those who receive extended attention at the site of trauma itself.

Medical care of the critically wounded depends, perhaps first and foremost, on the timely arrival of patients to hospitals. By the 1970s, a suggestive link between transport times to a medical facility and casualty survival rates emerged from patterns observed in the treatment of wounded Vietnam War military personnel. Although military technology was more dangerous than ever before, fewer soldiers were dying from their injuries. This paradoxical trend can be explained partly by the dramatic reduction in transit times of casualties: in the Vietnam War, wounded soldiers could expect to arrive at a medical facility

*To whom correspondence should be addressed:

Shaurya Taran

staran@gmail.com within one hour, while in the Korean War the average transit time for soldiers was five hours (1). Thus, it seemed that the quicker casualties arrived from the site of injury, the greater their chances were of a successful recovery. These findings also hold true in most other instances of general trauma. In very few cases it is preferable to administer intensive care at the site of the accident itself; speed in delivery seems to be the most important factor in patient recovery.

The fact that there is such a short period of opportunity for physicians to provide the appropriate care for trauma victims suggests the notion of a patient's "platinum minutes". If the medical needs of a trauma patient are met within these crucial minutes, then the likelihood of a patient surviving is dramatically increased. However, patients brought to medical facilities outside this window of time are much more likely to suffer from permanent complications, and are also at a greater risk of dying from their injuries. It seems, therefore, that a pre-clinical treatment approach in which the emphasis is on minimizing transit time between an accident site and a medical facility would ensure the best possible chances of recovery for trauma patients. This is precisely the idea behind the North American form of pre-clinical treatment. Termed "scoop and run," this strategy involves administering only Basic Life Support (BLS) at the trauma site before rushing patients to a hospital while they are still in their "platinum minutes". Although scoop and run is not always effective in specific situations, it remains arguably the best pre-clinical procedure in terms of its general suitability.

The benefits of the scoop and run form of treatment are perhaps best understood by examining death rates among soldiers in different wars. Physicians in the Korean War, for example, found that wounded soldiers who were brought quickly into the hospital for care had higher rates of survival than those who were attended to by primary caregivers on the battlefield itself. But perhaps a more compelling trend was found by 
contrasting death rates among combatants in the Korean War and Second World War. Analysts found that, among soldiers in critical condition, nearly twice as many soldiers survived their injuries in the Korean War than in the Second World War. One explanation is that the use of air transportation to move casualties during the Korean War dramatically reduced the transit time to a hospital, and consequently allowed physicians to operate more quickly on their incoming patients. The benefits of the scoop and run procedure can also be seen in comparing soldier mortality between the Second World War and the current wars in Afghanistan and Iraq. Only about $10 \%$ of soldiers wounded in the two wars today die from their injuries, as compared to $30 \%$ for soldiers in the Second World War (2). Although medical advancements are also largely responsible for this increase in survival rate among war casualties, the contribution of scoop and run to this success cannot be overlooked either.

Two other important points provide perhaps the most convincing evidence in support of the scoop and run procedure. The first comes from a study of over 12,000 trauma patients by J.S. Sampalis et. al. Researchers involved in the analysis found that for each minute trauma patients spent outside the hospital, the risk of mortality increased by 5\% (3). The second point is a direct comparison between transportation times of the North American and European procedures. In the European procedure (termed "stay and play") paramedics usually administer Advanced Life Support (ALS) at the site of trauma, which results in an average trip to the hospital of about 18.5 minutes. In the scoop and run procedure, where only Basic Life Support (BLS) is provided, emergency trips average 5 minutes less than when the stay and play procedure is employed. In medical emergencies where every minute without the appropriate attention of a physician can translate into the difference between life and death for a casualty, scoop and run clearly proves itself to be the most suitable form of pre-clinical treatment.

A final argument for the advantages of the scoop and run procedure over the stay and play approach can be made by considering the trends in numbers on what is called the Abbreviated Injury Scale. A casualty entering the Emergency Room is often rated on this scale according to the severity of his or her condition. The scale runs from 1 to 6 , with less serious injuries classifying as minor or moderate, and more severe conditions being critical or unsurvivable. Research shows that rapid delivery to the hospital from the site of an accident can actually lower the severity of a patient's condition on the Abbreviated Injury Scale (4). The differences are substantial, and include a drop in ratings on the Abbreviated Injury Scale from 4.9 (classified close to critical) to 3.4 (classified as serious). Of course, providing advanced care at a trauma site could also confer some benefits to patients, but these are not reflected by any major change in the numbers on the scale. This again suggests that a pre-clinical procedure in which the imperative is rapid delivery rather than comprehensive on-site care offers the greatest prospects of a trauma victim's full recovery.

In most instances, the scoop and run approach no doubt enjoys the greatest number of associated benefits; however, there are cases where an application of the less generally-suited stay and play practice would ultimately serve better. These cases include those where the number of casualties is too large to coordinate a successful rescue operation with limited evacuation assets (5). The 2004 Madrid train bombings, which resulted in 191 civilian deaths and 2050 injuries, illustrates one specific scenario where the stay and play approach was employed to good effect. Another case where the stay and play procedure is the only possible pre-hospital treatment strategy is when it is simply infeasible to employ the scoop and run approach. There can be several reasons why it is not possible to quickly, safely, and efficiently transport a casualty for comprehensive treatment at a medical facility; in these instances, the stay and play procedure can be used appropriately, provided that a strict protocol for the onsite treatment of trauma victims is followed. For this strategy to be appropriately conducted, paramedics must ensure that specific criteria unique to lengthy onsite care approaches are met within certain time windows.

These are very specific instances where stay and play is the only appropriate pre-hospital treatment strategy. Of course, it is clear that the need to employ stay and play arises more out of necessity in any given instance than practicality, and that the procedure would not serve well if a better alternative, namely scoop and run, was possible. Indeed, scoop and run remains the only procedure with the greatest number of benefits associated with its practice. In fact, so compelling was the evidence in its favour that The Australian Trauma and Emergency Services final report in 1999 specifically stated that not using scoop and run in emergency care was a pre-clinical system error (6). Therefore, in terms of the advantages it confers to trauma patients, the North American scoop and run procedure is arguably more effective in meeting the immediate needs of casualties than the corresponding European stay and play practice.

\section{REFERENCES}

1. Lerner EB, Moscati RM: The golden hour: Scientific fact or medical "urban legend"? Acad Emerg Med 2001; 8:758-760

2. "The recent emphasis on leaner, faster-moving military units 
added to the imperative to push surgical teams farther forward, closer to battle." Gawande A.Casualties of War - Military Care for the Wounded from Iraq and Afghanistan. (Accessed January 8,2009 at http://content.nejm.org/cgi/content/full/ $351 / 24 / 2471)$

3. Sampalis JS, Denis R, Lavoie A. Trauma care regionalization: a process-outcome evaluation. J Trauma 1999;46:565-79

4. Smith JP, Bodai BJ, Hill AS, Frey CF: Prehospital stabilization of critically injured patients: A failed concept. J Trauma 1985; 25: 65 .
5. Hodgetts, T, JMcManus, and MJ JHoejenbos. "Is there one optimal medical treatment and evacuation chain for all situations: "scoop-and-run" or "stay-and-play"." Prehospital and disaster medicine 23.4 (2008)

6. Review of Trauma and Emergency Services. Department of Human Services, 1999. (Accessed January 8, 2009, at http://www.health.vic.gov.au/trauma/review99/1c1.htm)

Shaurya Taran is a student in the first year of a three-year program in Anatomy and Cell Biology at McGill University. He is currently employed in a lab whose primary research focuses are axonal guidance and neuron migration in the Drosophila nervous system. His previous publications include a review article of the physiological benefits of yogic breathing techniques, which appeared in a 2008 edition of The Columbia Science Review. 